\title{
Frontières
}

\section{CAENEPEEL, Didier, La sédation continue en fin de vie. Enjeux éthiques, Montréal, Médiaspaul Canada, 2005, 240 p.}

\section{Jocelyne Saint-Arnaud}

Volume 18, numéro 2, printemps 2006

URI : https://id.erudit.org/iderudit/1073237ar

DOI : https://doi.org/10.7202/1073237ar

Aller au sommaire du numéro

Éditeur(s)

Université du Québec à Montréal

ISSN

1180-3479 (imprimé)

1916-0976 (numérique)

Découvrir la revue

Citer ce compte rendu

Saint-Arnaud, J. (2006). Compte rendu de [CAENEPEEL, Didier, La sédation continue en fin de vie. Enjeux éthiques, Montréal, Médiaspaul Canada, 2005, 240 p.] Frontières, 18(2), 83-84. https://doi.org/10.7202/1073237ar d'utilisation que vous pouvez consulter en ligne.

https://apropos.erudit.org/fr/usagers/politique-dutilisation/ 
CAENEPEEL, Didier

\section{La sédation continue en fin de vie} Enjeux éthiques

Montréal, Médiaspaul Canada, 2005, 240 p.

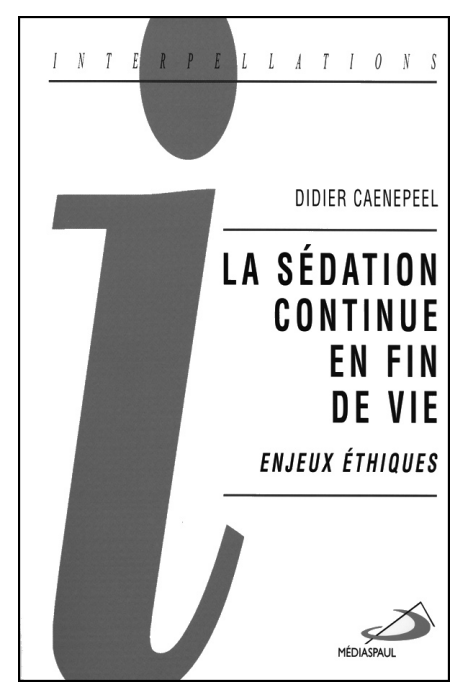

Ce petit livre de la collection Interpellations aux éditions Mediaspaul traite d'un sujet éthique peu discuté en dehors des milieux de la santé. La sédation continue en fin de vie fait référence à un sommeil induit par l'administration d'antalgiques en ayant pour but de soulager la douleur, voire la souffrance. Il existe en effet des situations, échappant au contrôle médical, dans lesquelles la souffrance est si grande que seule la narcose peut I'apaiser. Didier Caenepeel nous propose d'identifier les enjeux soulevés par une telle situation. Après avoir précisé la terminologie et la typologie relatives à la problématique soulevée, il établit une topolo- gie des lieux qui lui permet ensuite de décrire et de discuter d'un certain nombre d'enjeux éthiques suscités par la pratique de la sédation profonde autour des concepts d'euthanasie et de soins palliatifs.

Le premier chapitre présente différentes terminologies qualifiant I'intervention: sommeil pharmacoinduit, sédation active, profonde, complète ou continue, terminale ou palliative, mini-anesthésie, inconscience ou coma provoqués. Malgré cette diversité, il y a des convergences dans les définitions et l'auteur retient l'expression "sommeil induit" pour qualifier une intervention consistant à provoquer le sommeil au moyen d'analgésiques et en réponse à des indications médicales (p. 22). Faisant suite à la typologie, la topologie de la souffrance est identifiée à un espace relationnel défini par la triade: médecin, patient et entourage. Ces derniers deviennent partie prenante et éléments constitutifs de la problématique. Ils se rejoignent dans l'expérience de la douleur: le patient ne voulant pas souffrir, le médecin ne voulant pas laisser souffrir et l'entourage ne voulant pas voir souffrir. Ce noyau constitue le cœur et l'originalité de l'approche. $C^{\prime}$ 'est sous cet angle que seront abordés les liens entre sédation profonde et soins palliatifs au dernier chapitre de l'ouvrage.

Le deuxième chapitre utilise une approche heuristique pour préciser des repères éthiques portant sur la douleur et la souffrance et qui serviront d'assises pour discuter des enjeux éthiques de la sédation. D'une exploration sémantique des termes, I'auteur conclut à une difficulté de les définir et donc de les distinguer. Mais les différentes perspectives éclairant le phénomène, la douleur physique indique une perturbation de l'organisme, tandis que la souffrance est multidimensionnelle. Citant Ricoeur, la douleur est liée à un affect ressenti et localisé et la souffrance à des affects ouverts sur le rapport à soi et à autrui, ce qui rejoint la topologie proposée par l'auteur au chapitre précédent. Plus précisément, la phénoménologie de la souffrance, selon Ricoeur, se répartit sur deux axes, celui du rapport à soi et à autrui, d'une part, et celui de l'agir-pâtir, présent dans les définitions courantes, d'autre part. Dans cette perspective, la souffrance est une altération du rapport à soi et aux autres. Dans le rapport à soi, ce qui est atteint, c'est le rapport d'intentionnalité visant autre chose que soi; autrement dit, l'être souf- frant ne peut être centré que sur lui, le reste n'existe plus. Le rapport à l'autre est marqué par une crise de l'altérité sous l'égide de la solitude et de l'incommunicabilité. L'axe agir-pâtir se construit sur le premier axe et se résume à la diminution de la capacité d'agir. II exprime les modalités du souffrir. La souffrance totale fait naître le désir de mort.

Le but du troisième chapitre est de cerner les représentations que I'homme se fait de la mort, de même que les attitudes qui en découlent. Avec Ariès, I'auteur relate quatre périodes relatives aux attitudes face à la mort, de la mort apprivoisée du premier millénaire, à la mort interdite de la seconde moitié du XXe siècle jusqu'à maintenant, alors que le milieu hospitalier prend la mort en charge, modifie son lieu, ses modalités et sa chronologie. Les développements techniques et scientifiques font de la mort l'ennemi à vaincre et, dans ce contexte, la mort apparaît comme un échec. Passant brièvement sur les critères médicaux de la mort, l'auteur s'attarde à montrer comment la mort est souvent associée au sommeil, dans la mythologie, la Bible et les contes, notamment celui de la Belle au bois dormant qu'il analyse plus longuement. II en conclut que le sommeil induit artificiellement n'est pas de l'ordre du symbolisme, qu'il est plutôt «l'antichambre de la mort» (p. 123-124).

Suivant en cela les écrits étatsuniens sur la question, le quatrième chapitre examine les discours portant sur les liens entre sédation profonde et pratiques euthanasiques. II présente d'abord un survol historique de la signification du terme euthanasie en passant sous silence le suicide euthanasique pratiqué dans I'Antiquité. Dès cette époque, des tiers interviennent pour abréger les jours de personnes trop souffrantes, même si le terme euthanasie n'est pas utilisé pour désigner l'intervention. De plus, le XIXe siècle est présenté comme ayant donné lieu à un glissement dans la sémantique, au sens où ce serait à cette époque que le "prendre soin» aurait été introduit dans les discours pour favoriser une mort douce. Ce faisant, l'auteur ne rend pas justice à Francis Bacon qui, dans The Advancement of Learning paru en 1605 , enjoignait le médecin de mettre son art au service d'une bonne mort. La bonne mort concernait alors l'utilisation des ressources médicales en vue d'un soulagement de la douleur et un accompagnement du mourant en vue d'une préparation de nature spirituelle.
Le cœur du chapitre fait état de différents points de vue sur la sédation profonde au regard d'une pratique euthanasique. Selon un premier point de vue, la sédation profonde est une euthanasie déguisée puisqu'elle est suivie du retrait des moyens d'alimentation et d'hydratation (Orentlicher cité par Caenepeel, p. 150). Loewy est du même avis et considère que la distinction entre sédation et euthanasie est maintenue parce que la sédation profonde est légalement acceptée, ce qui n'est pas le cas de l'euthanasie, et parce qu'elle procure un certain réconfort au médecin et à son équipe (p. 152). Tännsjö, un philosophe suédois, y voit une solution de compromis, dans le cas où l'euthanasie ou le suicide assisté ne pourraient être pratiqués (p. 161). Par ailleurs, Cummins-Gauthier utilise le principe du double effet pour décrire les similitudes entre sédation profonde et euthanasie. Son but avoué est de faire la promotion de la légalisation de l'euthanasie et du suicide assisté (p. 153-154). Les auteurs Quill et Lo ont une position différente. Ils considèrent très complexe, cliniquement et éthiquement, la pratique de la sédation terminale et pour eux le principe du double effet ou la distinction entre intervention passive et active constituent des repères trop controversés pour servir de critère d'éthicité à la pratique de la sédation profonde. Ils considèrent que la pratique peut être moralement acceptable si elle répond au principe du respect de l'autonomie de la personne et du consentement libre et éclairé qui en découle et au principe de proportionnalité. À cela ils ajoutent les conditions suivantes:

- la reconnaissance que les soins palliatifs conventionnels sont insuffisants pour assurer le soulagement,

- le consentement informé du patient,

- la clarté dans le diagnostic et dans le pronostic,

- le recours à l'opinion d'un second professionnel et

- la documentation des cas (p.158-159).

Pour ces auteurs, la sédation est une mesure exceptionnelle. Elle est éthiquement acceptable, mais correspond à une solution du moindre mal.

De cette recension d'écrits, Didier Caenepeel conclut que les enjeux éthiques soulevés par la sédation terminale sont différents de ceux qui sont liés à l'arrêt des mesures d'alimentation et d'hydratation et qu'ils méritent un traitement diffé- 
rent. II s'en tient donc aux questions soulevées par la sédation, considérant l'arrêt de l'alimentation et de I'hydratation comme étant hors de son propos (p. 167). Or c'est principalement ce qui fait problème au regard de la pratique euthanasique, puisque la sédation, si elle est pratiquée selon les règles de l'art, n'entraîne pas la mort de la personne. C'est plutôt le fait que la personne endormie en permanence ne s'alimente plus qui cause sa mort. Quoi qu'il en soit, l'auteur évolue sous un registre différent de celui des auteurs qu'il cite dans la première partie du chapitre. De l'étude de leurs écrits concernés avant tout par l'éthique d'une intervention susceptible de hâter la mort, il conclut que, focalisant sur "l'échéance fatale, (ils) ne paraissent plus en mesure d'articuler la complexité de la problématique, notamment dans ses dimensions intersubjectives et symboliques» (p. 168). Selon lui, la problématique éthique est détournée et paralysée par un manque d'ouverture à une perspective plus large qui est celle des rapports relationnels de la triade patient-médecin-entourage. II reprend des commentaires de Pochard, Grassin et Hervé adressés à Quill et Lo critiquant la trop grande importance accordée au consentement libre et éclairé «négligeant de protéger les personnes concernées par les conséquences psychologiques de la mort du patient» (p. 171) et critiquant leurs lignes directrices comme étant fondées sur des données probantes et s'avérant trop théoriques pour être utiles dans la pratique. L'auteur semble faire sienne cette critique. Nous laissons au lecteur le soin de juger du fondement de cette critique, compte tenu du fait que les auteurs Quill et Lo (Lo est un philosophe et non un médecin comme le laisse entendre l'auteur) 1) mentionnent aussi l'importance de respecter le principe de proportionnalité (et non seulement le principe du respect de l'autonomie de la personne); 2) leurs directives n'ont rien à voir avec des données probantes, si c'était le cas, elles auraient été tirées de données empiriques; et 3) loin d'être théoriques, elles sont très concrètes. Le lecteur peut s'en assurer par luimême puisqu'elles sont citées plus haut.

Le chapitre se termine sur la réponse que Pie XII avait faite aux anesthésistes sur la question en 1957. Parlant au nom de l'Église, le pape, dans un discours très cohérent, avait indiqué que la sédation profonde ou narcose est moralement permise pourvu que la personne ne soit pas privée de remplir ses devoirs envers les autres et s'il n'existe pas d'autres moyens thérapeutiques. Si la personne a rempli ses devoirs, que les indications médicales le suggèrent, que les doses respectent la pratique admise, que la durée et l'intensité sont mesurées et que le patient y consent, la sédation profonde est moralement acceptable, même si elle implique un état d'inconscience (p. 179).

Dans le cinquième chapitre, Caenepeel réintroduit, à juste titre, la problématique de la sédation profonde dans la perspective plus large des soins palliatifs. II souligne le paradoxe lié au fait que la sédation profonde implique une perte de conscience, alors que, depuis Cicely Saunders (son nom est mal orthographié dans le livre), les soins palliatifs sont axés sur les relations humaines et les soins de confort conçus selon une approche globale de soin. De ce dernier point de vue, il serait impératif de maintenir la conscience jusqu'à la fin. Peut-on parler d'accompagner une personne endormie? L'auteur répond affirmativement à cette question. L'accompagnement est possible si l'accompagnant est celui qui témoigne d'une volonté exprimée dans un partage authentique, alors que la parole pouvait encore être échangée. La relation éthique se développe dans un espace intersubjectif. C'est dans une telle relation que la question de la sédation terminale (comme tout type de sédation d'ailleurs) peut être abordée. Mais il subsiste une ambiguïté, du point de vue de la pratique, à la position de l'auteur: sont-ce les volontés manifestées antérieurement par la personne endormie qui doivent être respectées ou les volontés de la triade patient-médecin-entourage? Loin de donner priorité aux désirs du patient, l'auteur recommande que les divers acteurs de la triade "puissent, dans un climat de réciprocité, faire part ouvertement de leurs volontés». Sont-ce les volontés du patient ou celles de la triade qui seront déterminantes dans la décision d'appliquer la sédation? ॥ semble difficile pour l'auteur de privilégier le respect de l'autonomie de la personne, puisque, dans la triade, le patient ne constitue qu'une voix parmi les autres, et qu'il remet en question la pertinence d'appliquer la règle du consentement libre et éclairé, comme cela a été mentionné au chapitre précédent. Et sur quelles bases philosophiques respecter I'humanité dans la personne endormie, si le principe du respect de l'autonomie de la personne n'a pas été retenu dans l'analyse?
En conclusion, le livre comporte des forces et des faiblesses. II s'agit d'un mémoire de maîtrise qui semble avoir été publié comme tel, on retrouve le terme "mémoire" à quelques reprises dans le texte. L'auteur aurait eu intérêt à resserrer son analyse et à traiter plus à fond de ce qui est central à sa problématique. Par exemple, jamais il ne présente une analyse critique de la doctrine du double effet à laquelle de très nombreux auteurs cités font référence. Continuellement dans le texte, on peut lire que le propos présenté demanderait une analyse plus approfondie, sans que jamais l'auteur juge approprié de le faire.

Les forces de l'ouvrage sont de susciter la réflexion sur des enjeux actuels et peu discutés dans les écrits, de relater des références pertinentes sur le sujet traité, les notes sont très riches à cet égard -, d'articuler son argumentation et son analyse personnelle autour de la triade patient-médecinentourage comme lieu de la souffrance. À cet égard, le deuxième chapitre de l'ouvrage, notamment dans sa réflexion sur la douleur et la souffrance selon Ricoeur, est très pertinent pour traiter de cet épineux sujet sans tomber dans une approche dualiste de l'être humain et de l'intervention sur l'être humain. La philosophie de base développée dans l'ouvrage constitue le fondement de toute approche globale de soins.

Jocelyne Saint-Arnaud 